\title{
VSA-TOOL: A TOOL FOR DATA VISUALIZATION IN SEQUENCE ALIGNMENT
}

\author{
Li-Yeh Chuang ${ }^{1}$, Cheng-Hong Yang ${ }^{2}$, ChaO-Ching Chang ${ }^{2}$, Wen-Shyong Tzou ${ }^{3}$, Li-Cheng Jin $^{2}$ \\ ${ }^{1}$ Dept of Chemical Eng., I-Shou University, Kaohsiung \\ ${ }^{2}$ Dept of Electronic Eng., National Kaohsiung University of Applied Sciences, Kaohsiung \\ ${ }^{3}$ MedicoGenomic Research Center \& Biotechnology, Kaohsiung Medical University, \\ Kaohsiung, Taiwan
}

\begin{abstract}
Sequence alignment is a fundamental and important tool for sequence data analysis in molecular biology. Many applications in molecular biology require the detection of a similarity pattern displayed by a number of DNA and protein sequences. Visual front-ends are useful for an intuitive viewing of alignment and help to analyze the structure, functions, and evolution of the DNA and protein. In this paper, we designed and implemented an interactive system for data visualization in DNA and proteins, which can be used in determining a sequence alignment, similarity search of sequence data, and function interference. Experimental results show that a user can easily operate the system after one hour's practice on the proposed system, which provides a clean output. easy identification of similarity and visualization of alignment data.
\end{abstract}

Biomed Eng Appl Basis Comm, 2004 (April); 16: 68-72.

Keywords: data visualization, sequence alignment, DNA, protein.

\section{INTRODUCTION}

Sequence analysis has become an important field in molecular biology because the sequence of a DNA or protein carries a lot of information about its biomolecular function and history [1]. In general, scientists assume that similar sequences of DNA or proteins hold a similar function or structure, and that they have a similar evolutionary history. Thus, it is not surprising that the multiple sequence alignments of bio-molecules have become an essential tool in bioinformatics. Procedures relying on sequence comparison are diverse and range from database

Recejved: Dec 15, 2003; Accepted: Jan 29, 2004

Correspondence: Cheng-Hong Yang, Professor

Dept of Electronic Eng., National Kaohsiung University of Applied Sciences, Kaohsiung, Taiwan E-mail: chyang@cc.kuas.edu.tw searches to structure prediction. Sequences can be compared two-by-two by scouring databases for homologues, or they can be multiply aligned to visualize the effect of evolution across a whole genome or protein family. The development of accurate, reliable and visualized multiple alignment programs capable of handling the divergent sets of data is therefore of major importance.

There are many ways to align two sequences, including dot matrix analysis, dynamic programming algorithms and word or k-tuple methods [2]. Two famous alignment methods for comparing two sequences are the Smith-Waterman or DynamicProgramming alignment (guarantees to find an optimal solution for two given sequences and a given scoring scheme), or the Blast alignment (not guaranteed to be optimal, the result is generally much shorter than that of the previous method). Moreover, a lot of alignment methods were developed to align multiple sequences. Some are progressive methods (ClustalW, PILEUP), iterative methods (genctic algorithm and hidden 
Markov model) and statistical methods (expectation maximization algorithm and Gibbs sampler) [2-3].

The literature and Internet present several examples of software for sequence alignment. Some of these software tools are WISE, COMBAT, and EMBOSS and loadseq, ClustalW, DCA, PIMA, DIALIGN 2, treealign, ComAlign, BOXSHADE, MVIEW, plotcon, and cons for pairwise comparisons and multiple alignment comparison [4].

Sequence data can be expressed either verbally or graphically. Thus, the results of biological sequence comparison (DNA or protein) are displayed in text or a variety of graphs to explain their similarities and differences [5-8]. The expressed data is usually difficult to interpret and not user-friendly. Thus, in this study, we present an interactive system for biological sequence alignment. Compared with two other alignment software tools in the literature, GeneDoc and BioEdit [9], the proposed system improves user friendliness, generalizes the sequence pattern, and uses various figures of expression, which make the data easily understandable and interpretable.

\section{SYSTEM DESIGN}

Over the past ten years, biological sequence analysis has become an important research area in biological studies. Without a doubt, it will be a challenge for the future bio-medical scientist to combine biological, medical, and computer expertise to investigate the information contained in genes, to understand the structure and functions of genes, and the role of different biophysical and physiomedical conditions.

The calculation of alignment is one of the most basic tasks when analyzing biological sequences. For instance, any database search involves an alignment step. Algorithms calculating alignments come in different flavors: routines to calculate global or local alignments, routines to calculate pair-wise or multiple alignments, routines to calculate strictly optimal or only nearly optimal (heuristic) alignments. Furthermore, algorithms are often tailored to specific situations. For instance, alignment of a genomic sequence with an mRNA-sequence is a different task and is solved differently than aligning two genomic sequences.

\subsection{Data Visualization}

The most challenging aspect of visualization is to express the large amounts and multiple dimensions of data on a computer screen. One of the difficulties is that data are $\mathrm{N}$-dimensional $(\mathrm{N} \geqq 3)$, however a computer screen can only reveal two- or three- dimensional data. How to map $\mathrm{N}$-dimensional data into $2 \mathrm{D}$ or $3 \mathrm{D}$ space is the first problem that has to be solved for data visualization. Another difficulty for visualization design is that we don't really know how humans extract information from pictures or image. Even though there are theories of visualized recognition that can be used in some special fields, these theories actually are not very useful for visualization design. Moreover, it seems that visualization design is related to domain-specifics. It usually shows different results based on the definition of the problem, purpose, the background of people, experience and visual metaphors. Therefore, it is difficult to develop a formal and general rule for data visualization. At present, most scholars develop visualization technologies according to their experience and trial-and-error methods. We would like to point out that an efficient visualization system must correspond to basic design rules. Most importantly we have to consider the problem's definition and purpose, as well as the data's semantic meaning. The same data set has to have different visualization expressions based on different purposes, in much the same way as different persons will have different points of view about the same visual image.

If an image contains too much data, the results of visualization will lead to an inability to fully reveal and comprehend information due to the fact that the visual image appears to be cluttered. Therefore, simplification of the complex data is necessary. Simplification of the data, as well as retention of the data character, is very important for visualization. In general, the simplification technology for complicate data contains three simplifications: simplification of data amount, simplification of data dimension, and decrease of the data image directly. The data amount is simplified by using clustering or networks to decrease M1 sets of data into $M 2$ sets $(M 1 \geqq M 2)$. Simplification of data dimension can be achieved by using principal component analysis or networks to decrease $\mathrm{N} 1$ dimensions of each data set into $\mathrm{N} 2$ dimensions $(\mathrm{N} 2=$ 2,$3 ; \mathrm{N} 1>\mathrm{N} 2$ ) for conveniently obtaining data figures. Also, anti-aliasing technology is used to decrease the data image and keep the important visual patterns of the image.

Benchmarking and verification of visualization techniques are lacking formal and general design rules. The major reason for this is the fact that visualization has to depend on a subjective point of view by the user; it is difficult to objectively define the quality and quantity of information, which is absorbed by the users from a data image. In the past, many scholars developed various visualization techniques. However, these are only applied in specific fields and have a special function. They are lacking formal discussion and we cannot be sure if these methods can be 
generalized. Therefore, designing and standardizing objective evaluations for benchmarking data sets is a worthy investigation point.

\subsection{Software Functions}

The sequence analysis process used is schematically summarized in Figure. 1. After the data input into the system, sequences that are to be compared are selected. These sequences are checked for alignment and the results can either be used as are, or can be compared to a web-based data bank. A further step visualizes the results and verifies them before the final data is graphically presented.

There are six major functions incorporated in this system:

FILE: file operations include: creating, opening, saving, and submitting. The formats used are txt, doc, and aln (aln was used in ClustalW).

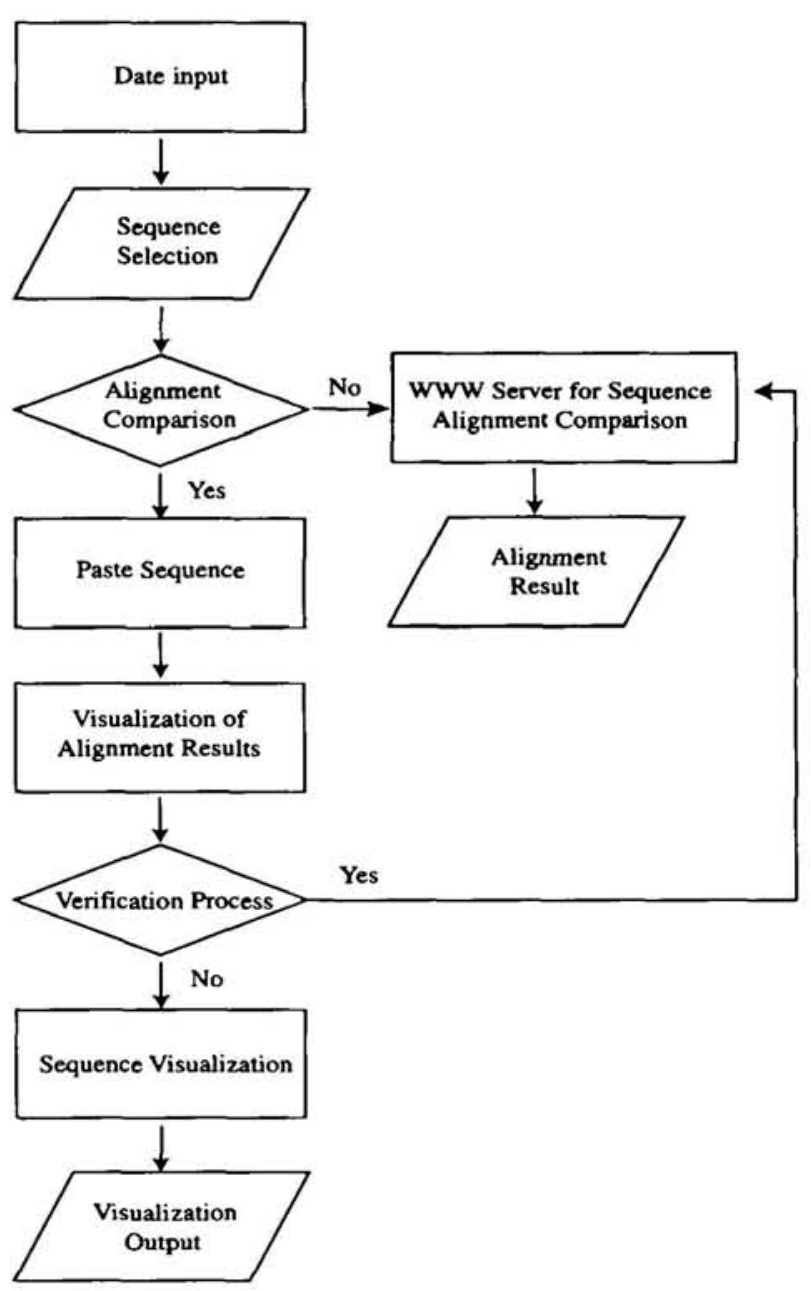

Fig 1. Workflow diagram. Workflow for sequence analysis processes, with VSA-Tool.
$E D I T$ : several editing functions, such as creating, deleting, shifting, are provided to help users to edit the sequence. A user can select the desired biological sequence, then update, insert, or delete the sequence. After the above operation, the data can be sent and compared to a web server again. This enables the user to compare the modified sequence with the previous sequences.

ALIGNMENT METHOD: The alignment method can be used in conjunction with the WWW to compare DNA and protein sequences, and also in conjunction with other methods, including BLAST, ClustalW, BCM_Launcher, Multiple Alignment, FGENESH, GeneID (v.1), GeneMark.hmm (v. 2.2a), Genie, GENSCAN, GenView, Grail II, GrailEXP-Perceval (v.3.0), MZEF, AAT, GeneBuilder, GrailEXP, CluastalW, and ExPASy (David, 2001). Thus, users have a choice of various alignment methods, which can be selected to facilitate comparison.

DATA VISUALIZATION: the system provides a variety of graphic visualizations, including line graphs, circles, histograms, text, and degrees of similarity (entirely or partially color-coded or represented in dot maps).

HELP: introduces the purpose of the system, its operational methods, content of input data, and output results.

The system also contains auxiliary functions that simplify similarity comparisons. For example, if the similarity comparison of all samples leads to a result that still seems too cluttered, a partial comparison of selective samples can be conducted (Figure. 2-6). Additionally, the results of the sequence alignment obtained by different alignment methods can also be compared. Figure. 2-6 demonstrate some of these auxiliary functions.

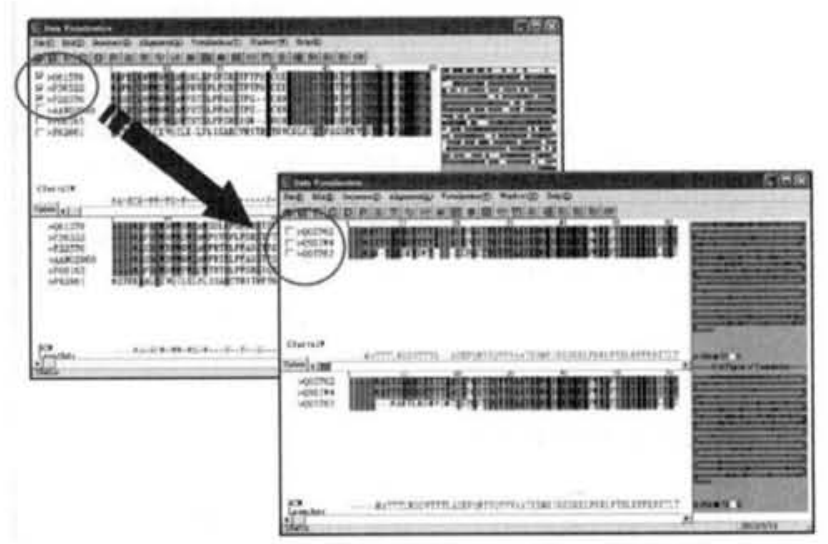

Fig 2. Refined data analysis of randomly chosen sequencing data. 


\section{RESULTS AND DISCUSSIONS}

The system is implemented using Microsoft Visual Basic 6.0, Windows version. Sequence analysis encompasses the use of various bioinformatics methods to determine the biological function and/or structure of genes and the proteins they encode.

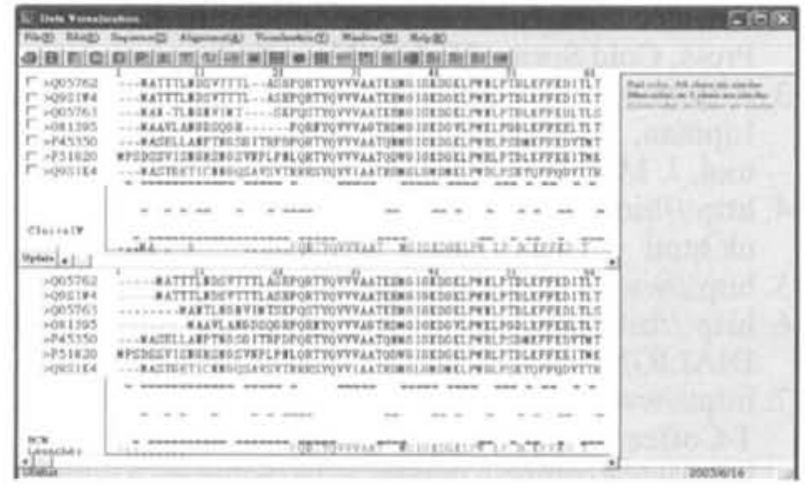

Fig 3. Comparison of amino acid groupings.

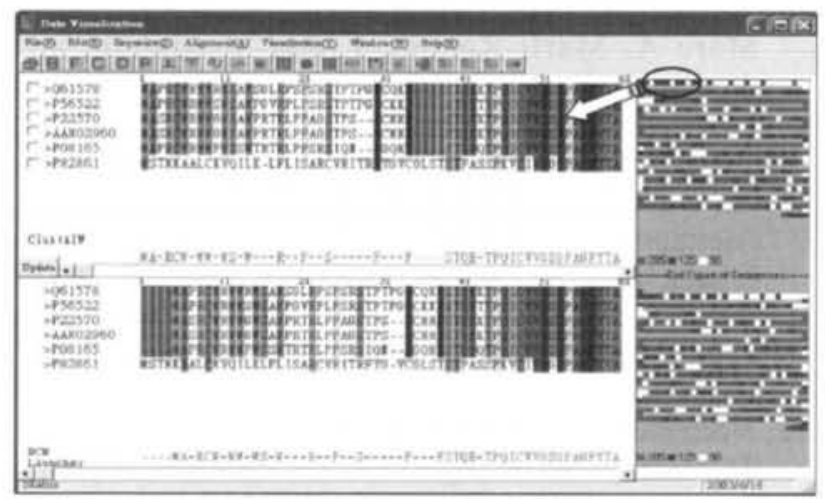

Fig 4. User-defined display segmentation (graphically represented here as a dot sequence).

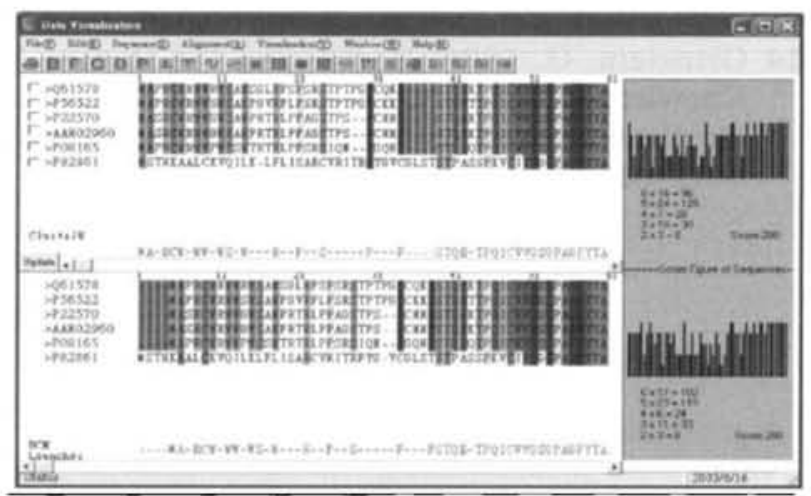

Fig 5. Similarity of amino acids grouping $s$ shown as histograms.

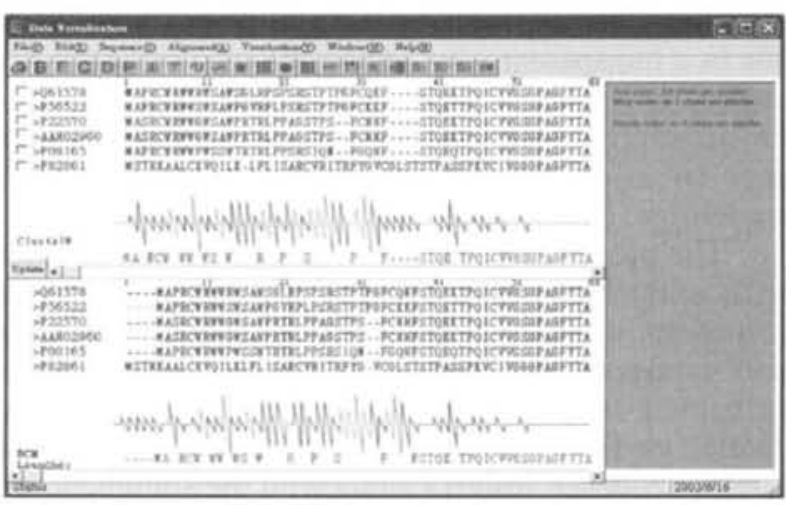

Fig 6. Wave representation of sequence alignment.

Several alignment methods and tools, which calculate the best match for the selected sequences, and line them up so that identical sequences, similarities and differences can be seen, were designed and implemented to help determine the protein coding regions of a DNA molecule or protein sequence [1011]. Many packages provide powerful alignment algorithms, such as T-Coffee [12] and ClustalW [13], but they still need to be guided by human insight. Therefore, it is necessary to integrate the sequence alignment visually [14]. In the proposed system, regions where both alignments coincide are colorcoded to visualize the local agreement between the two alignments, and able to identify regional similarities and differences of the alignments.

The proposed system combines the following critical features: (1) ability to visualize results of two different alignment methods simultaneously, (2) an annotation file for the base sequence, in txt, doc, and aln (ClustalW) format, (3) ability to analyze three different types of sequences: randomly generated sequence, customizable input sequence, and programmed default sequence, (4) the use of a continuous curve to represent the level of identification, (5) a user-friendly interface and operations that can easily be modified by inserting or deleting a sequence, (6) option of inserting new sequences, (7) option of editing a sequence, and a feature for real-time re-calculation of alignment, (8) a new line for sequence annotation purposes, (9) an alignment score view in the right panel beside the sequence alignment window, and (10) a summary line that describes the chemical properties of amino acids (3 types: hydrophobic, charged and polar).

Multiple alignments constitute an extremely powerful means of revealing the constraints imposed by structure and function on the evolution of a protein family. But, when the data is heterogeneous, knowing what is right and what is wrong becomes an art. Addressing that type of question will be difficult and 
essential. The appropriate method would have to do this in a transparent way, letting the user visualize and control every bit of extra information that goes into this alignment. This ideal method should also allow the user to inject into his model some of his own knowledge. Doing so should be made an easy task.

The proposed system can be used in combination with existing WWW servers to compare protein sequences, or through the ACNUC server for nucleic acid sequences. Sequences can be entered by a user or extracted from SWISS-PROT, NCBI, GenBank, EMBL, or HOVERGEN. The system is also able to display sequence features along with the alignment.

Data visual front-ends of biological sequences are necessary to make the process of viewing alignments intuitive and to facility discovery of conserved sequences for functionally significant regions. Due to the fact that the data contained in DNA or protein sequences are extremely large, a data mining technology should combine computer algorithm-based and visual mining. Information gathered by these means greatly enhances a scientist's understanding of DNA and protein sequences.

\section{CONCLUSION}

In this paper, we proposed an interactive graphical system, which can be used to visualize biological sequence alignments. Sequences can be entered by a user or extracted from a database. By clicking on a block, the user can visualize the corresponding local alignment. Visual front-ends are useful for an intuitive viewing of alignment and help to analyze the structure, functions, and evolution of a biological molecule. The proposed system can be used in conjunction with the WWW to compare DNA and protein sequences. Experimental results shows that a user can easily operate the system after one hour's practice on the system, which provides a clean output, easy identification of similarity and visualization of alignment data.

\section{ACKNOWLEDGMENT}

This work was supported by the National Science Council (NSC) R.O.C., under contract NSC 92-2515S-214-002.

\section{REFERENCES}

1. Cappey, C., Danckaert, A., Dessen, P., and Hazout, Mash, S. 1991. An interactive program for multiple alignment and concensus sequence construction for biological sequences. Computer Application in the Biosciences, CABIOS. 7:195-202.

2. David, W. M. 2001. Bioinformatics: Sequence and Genome Analysis, Cold Spring Harbor Laboratory Press, Cold Spring Harbor, New York.

3. Altschul, S. F., Gish, W., Miller, W., Myers, E. and Lipman, D. 1990. A basic local alignment search tool. J. Mol. Biol. 215: 403-410.

4. http://bioweb.pasteur.fr/seqanal/alignment/introuk.html

5. http://www.ebi.ac.uk/clustalw, ClustalW

6. http://bibiserv.techfak.uni-bielefeld.de/dialign, DIALIGN

7. http://www.ch.embnet.org/software/TCoffee.html, T-Coffee

8. http://www.compbio.dundee.ac.uk/Software/Alscript/al script.html, Alscript

9. http://www.ebi.ac.uk

10. Valentin A. Ilyin, Ursula Pieper, Ashley C. Stuart, Marc A. Marti-Renom, Linda McMahan, and Andrej Sali, 2003, ModView, visualization of multiple protein sequences and structures, Bioinformatics 2003 19: 165-166.

11. Chris Mayor, Michael Brudno, Jody R. Schwartz, Alexander Poliakov, Edward M. Rubin, Kelly A. Frazer, Lior S. Pachter, and Inna Dubchak, 2000, VISTA : visualizing global DNA sequence alignments of arbitrary length, Bioinformatics 2000 16: 1046-1047.

12. Notredame, C., Higgins, D. G., Heringa, J. 2000. TCoffee: A novel algorithm for multiple sequence alignment. J. Mol. Biol. 302: 205- 217.

13. Thompson, J.D., Plewniak, F., Poch, O. 1999. A comprehensive comparison of multiple sequence alignment programs. Nucleic Acids Res. 27: 26822690.

14. Grinstein, G. 1996. Harnessing the Human in Knowledge Discovery. Proceedings of the second International Conference on Knowledge Discovery and Data Mining. 384-385. 